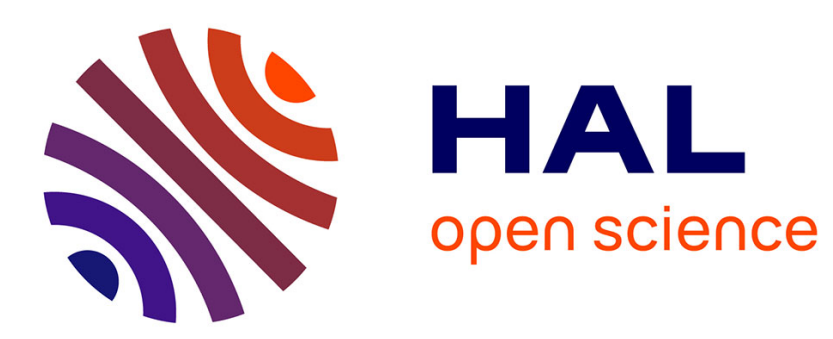

\title{
SYSTÈME DE MISE EN FORME DU CHAMP ACOUSTIQUE "ARTEMIS"
}

Grégory Chatel, J. Pascalini, J. Danthez, A. Roure

\section{To cite this version:}

Grégory Chatel, J. Pascalini, J. Danthez, A. Roure. SYSTÈME DE MISE EN FORME DU CHAMP ACOUSTIQUE "ARTEMIS". Journal de Physique IV Proceedings, 1992, 02 (C1), pp.C1-483-C1-486. 10.1051/jp4:19921104 . jpa-00251058

\section{HAL Id: jpa-00251058 https://hal.science/jpa-00251058}

Submitted on 1 Jan 1992

HAL is a multi-disciplinary open access archive for the deposit and dissemination of scientific research documents, whether they are published or not. The documents may come from teaching and research institutions in France or abroad, or from public or private research centers.
L'archive ouverte pluridisciplinaire HAL, est destinée au dépôt et à la diffusion de documents scientifiques de niveau recherche, publiés ou non, émanant des établissements d'enseignement et de recherche français ou étrangers, des laboratoires publics ou privés. 


\title{
SYSTÈME DE MISE EN FORME DU CHAMP ACOUSTIQUE "ARTEMIS"
}

\author{
G. CHATEL, J.P. PASCALINI, J.M. DANTHEZ* et A. ROURE** \\ SECAV, 6 Bld de Brazza, F-13008 Marseille, France \\ ${ }^{*}$ DCN Toulon CERDAN, BP. 77, F-83800 Toulon Naval, France \\ ${ }^{* *}$ CNRS LMA, 31 Chemin Joseph Aiguier, F-13009 Marseille, France
}

\begin{abstract}
Résumé - Le système "ARTEMIS" est un équipement de pilotage de sources acoustiques permettant la production de champs sonores ayant des caractéristiques prédéterminées.

La mise en forme de champs acoustiques, obtenue par cette méthode dans des milieux air et eau, est présentée et interprétée.

Le système "ARTEMIS" est appliqué à la mesure, par méthode réciproque, du rayonnement acoustique champ libre d'une structure immergée dans une lame d'eau. Les résultats sont validés à partir de mesures comparatives directes en eau profonde mettant en oeuvre une antenne hydrophonique.
\end{abstract}

Abstract - ARTEMIS is a new equipment used to compute free-field transfer function by piloting an active acoustic source array.

In this paper, we presented the experimental results obtained on the compute of the acoustic field in air and water. The experimetal validation of the method is made by comparison between the real free-field measurement in depth water and the reciprocal measurement in a water bounded medium with "ARTEMIS" equipement.

\section{1 - INTRODUCTION}

L'antenne d'émission comprend plusieurs sources acoustiques pilotées par un synthétiseur numérique programmable multi-voies dont les signaux ont été déterminés par des algorithmes de type L.M.S.

Dans des milieux confinés, oû la présence de frontières est génératrice d'échos parasites, le système "ARTEMIS" est conçu pour la création d'un champ acoustique identique à celui que donnerait en champ libre, une source acoustique ponctuelle monopolaire.

Le système permet la mise en forme d'ondes spériques ou d'ondes planes dans un volume d'observation.

Lorsque la structure testée est immergée dans le volume d'observation, une méthode de mesure réciproque, permet de déterminer le rayonnement acoustique champ libre de la structure soumise à une excitation par des forces ponctuelles.

\section{2 - MISE EN FORME DU CHAMP ACOUSTIQUE}

La mise en forme du champ acoustique consiste à créer dans un domaine $\Omega$ entouré d'une frontière $\Sigma$, un champ de pression acoustique aussi proche que possible de celui que fournirait une source ponctuelle en champ libre. 
On dispose pour cela de $\mathrm{N}$ sources acoustiques. alimentées chacune par une tension $\mathrm{U}_{\mathrm{j}}(\mathrm{f})$. La méthode consiste à prendre en considération $M$ points d'observation positionnés à l'intérieur de la zone dans laquelle on souhaite générer le champ acoustique $\mathrm{C}_{\mathrm{i}}(\mathrm{f})$ souhaité et à mesurer successivement les $\mathrm{N} \times \mathrm{M}$ fonctions de transfert $\mathrm{H}_{\mathrm{ij}}(\mathrm{f})$ entre la pression acoustique mesurée au point $i$ et la tension appliquée à la source $\mathrm{j}$. La solution est classique et s'exprime sous forme matricielle par :

$$
U=\left(H^{*} \cdot H\right)^{-1} . H^{*} . C
$$

avec l'indice * signifiant transposé-conjugué

Par transformées de Fourier inverses, on trouve les signaux temporels périodiques $U_{j}(t)$ à

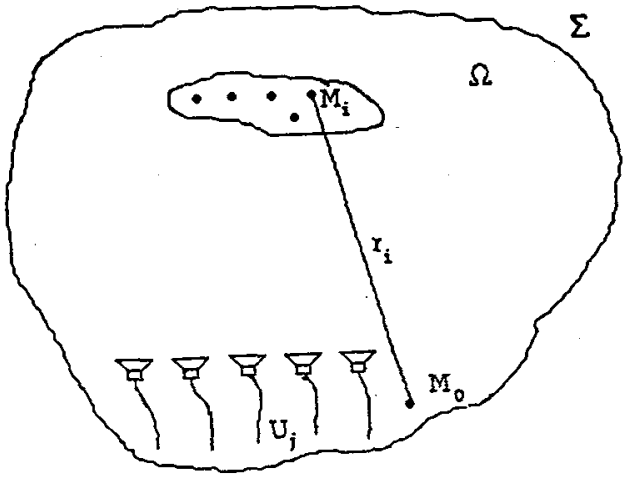

Figure 1 : Principe de la méthode appliquer à chaque source pour produire le champ désiré.

On choisit en général $C_{i}(f)=i \omega p Q(f) \exp \left(-i k\left|M_{0} M_{i}\right|\right) /\left(4 \pi\left|M_{0} M_{i}\right|\right)$ qui correspond à la pression produite en $\mathrm{M}_{\mathrm{i}}$ par une source ponctuelle placée en Mo et de débit volumique $Q(f)$.

\section{3 - CARACTERISTIQUES DU SYSTEME ARTEMIS}

"ARTEMIS" est un système, Hardware et Software, de pilotage d'antenne de sources acoustiques permettant la production de champs sonores ayant des caractéristiques prédéterminées.

Il est couplé à un analyseur et constitué de deux parties distinctes :

- un synthétiseur numérique programmable multivoies,

- un logiciel de pilotage implanté sur Ordinateur H.P. 9000.

Le synthétiseur se compose de douze voies indépendantes dans lesquelles peuvent être chargés les échantillons numériques des signaux à engendrer. Ces échantillons, au nombre de 2048 par voie, sont calculés par le logiciel. Envoyés sur des convertisseurs digitauxanalogiques à une cadence fournie par une horloge externe, ils fournissent aux amplificateurs des sources les signaux analogiques qui permettront d'obtenir le champ souhaité.

\section{4 - METHODE POUR LA MESURE DE FONCTIONS DE TRANSFERT MECANO. ACOUSTIQUE CHAMP LIBRE}

Les fonctions de transfert mécano-acoustique champ libre sont obtenues lorsque la structure testée est immergée dans un volume infini.

La méthode utilisée par le système "ARTEMIS" consiste à créer en bassin, à l'intérieur du volume de fluide ou sera ensuite immergée la structure en essais, le même champ acoustique que celui que l'on aurait en champ libre.

Ces conditions permettent d'obtenir une relation de réciprocité sur les fonctions de transfert champ libre :

$$
\mathrm{P} / \mathrm{F}=\gamma / \mathrm{Q}
$$

dans laquelle $\gamma$ est l'accélération vibratoire mesurée sur la structure, et $Q$ le débit d'accélération volumique du monopôle équivalent à l'antenne de sources. 


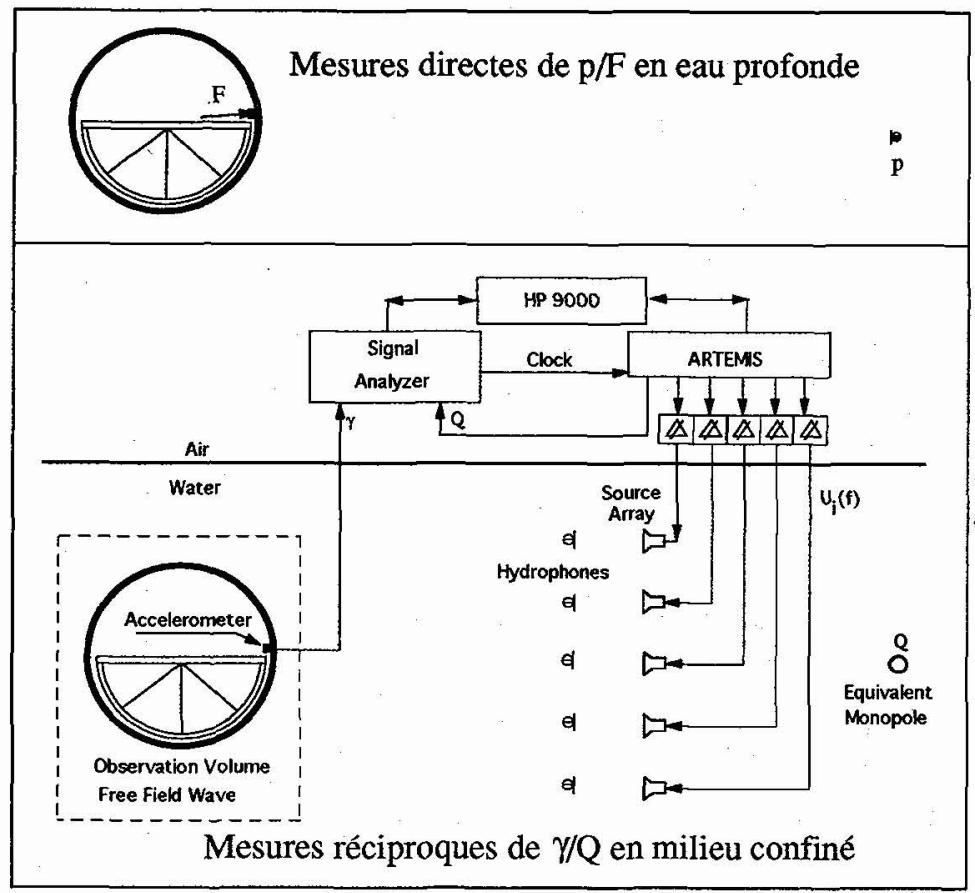

Fig. 2 : Mesure de fonctions de transfert $P / F$ champ libre

\section{5 - GENERATION D'UN CHAMP LIBRE DANS UNE ZONE D'OBSERVATION}

Le contrôle du champ acoustique réalisé dans un volume d'observation par le système ARTEMIS, a été effectué avant immersion de la structure testée.

Le champ de pression obtenu est systèmatiquement comparé en module et en phase au champ acoustique que produirait en champ libre la source virtuelle dont l'emplacement a été préalablement retenu par l'opérateur.

Les analyses montrent que la mise en oeuvre du système ARTEMIS permet d'obtenir dans l'ensemble du volume d'observation un champ sensiblement identique à celui d'une onde sphérique, ou d'une onde plane.

Fig.3 : Champ acoustique dans le volume d'observation 3.a - produit par une source 3.b - produit par ARTEMIS

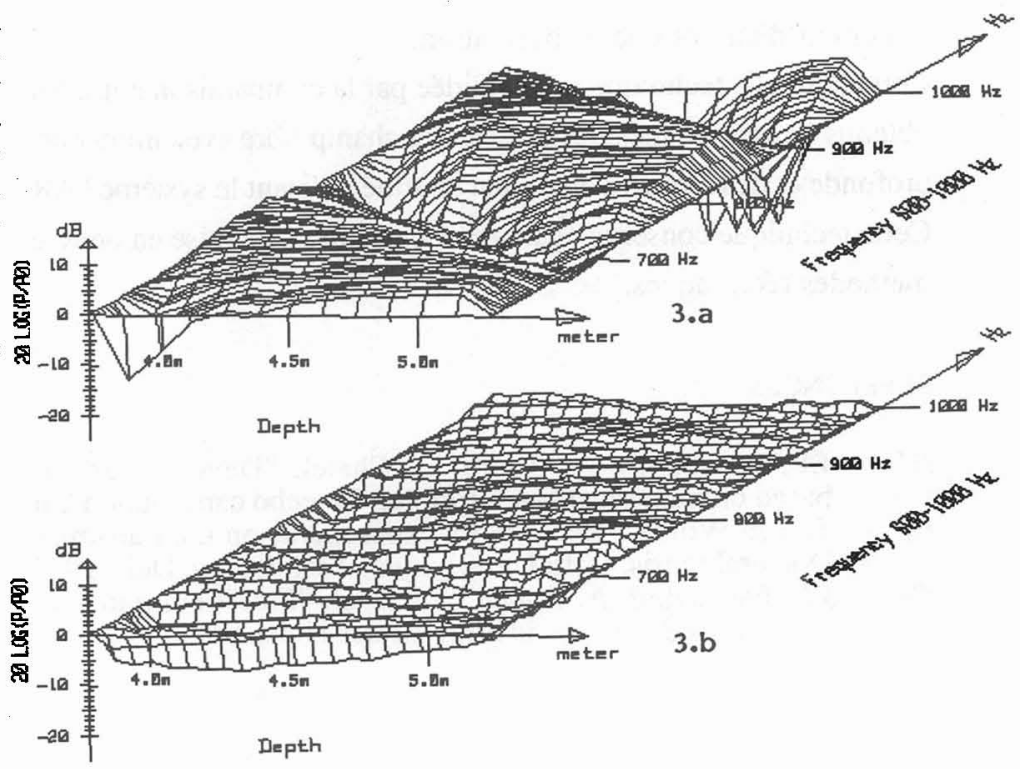




\section{6 - MESURES COMPARATIVES DES TRANSFERTS CHAMP LIBRE}

Les transferts mécano-acoustiques $\mathrm{P} / \mathrm{F}$ champ libre d'une structure métallique cylindrique de 3 mètres de long et de 1,20 mètre de diamètre, ont été mesurés par méthode réciproque en utilisant le systeme "ARTEMIS" (Figure.2).

Afin de valider les résultats, il a été ensuite procédé à la mesure directe de ces mêmes fonctions de transferts après avoir immergé la structure testée à 11,50 mètres de profondeur et en utilisant une antenne d'hydrophones.

On observe sur la figure 4, que le transfert champ libre obtenu par méthode réciproque avec le système "ARTEMIS", est sensiblement identique à celui mesuré par méthode directe sur la bande de fréquences adaptée à l'antenne.

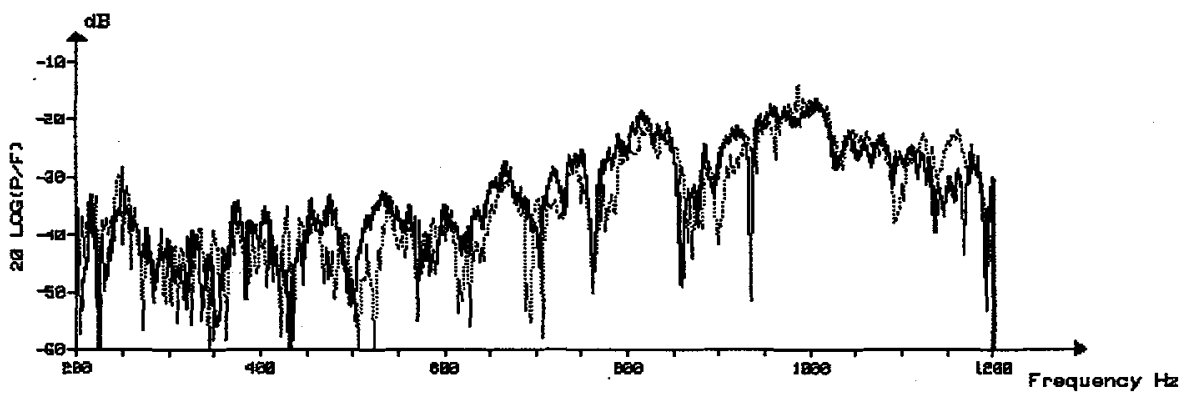

Figure 4 : Comparaison des fonctions de transfert P/F champ libre :

- trait plein : mesure directe, structure en eau profonde

- trait pointillé : mesure réciproque, en milieu confiné avec le système "ARTEMIS"

\section{7 - CONCLUSION}

Le système "ARTEMIS" a été développé pour mesurer des fonctions de transfert champ libre lorsque les essais sont effectués en présence d'échos parasites.

Le pilotage de l'antenne de sources permet la mise en forme du champ acoustique désiré à l'intérieur d'un volume d'observation.

Cette nouvelle technique a été validée par la comparaison entre les résultats des transferts obtenus à partir de mesures directes en champ libre avec immersion de la structure en eau profonde et des mesures en milieu confiné utilisant le système "ARTEMIS".

Cette technique conserve également les facilités de mise en oeuvre liées à l'utilisation des méthodes réciproques.

\section{REFERENCES}

11 J.P. Pasqualini, A. Roure, G. Chatel, "Development of an underwater array based on numerical beam forming for echo cancellation", Inter-Noise 88, 163-166

121 T. Ten Wolde, "Reciprocity experiments on the transmission of sound in ships" Doctoral thesis, Delft University of Technology, Delft 1973

13/ J.P. Pasqualini, A. Roure, G. Chatel, "Free-field transfer function measurement using an active acoustic array", Inter-Noise 91. 